\section{Wie werden interstitielle Lungenerkrankungen in Deutschland diagnostiziert?}

Ergebnisse des wissenschaftlichen Registers zur Erforschung von interstitiellen Lungenerkrankungen („Fibroseregister“) der WATL

\author{
How are interstitial lung diseases diagnosed in Germany? \\ Results of the scientific registry for the exploration of interstitial lung diseases \\ ("Fibrosis registry") of the WATL
}

\section{Zusammenfassung}

Zur Erfassung interstitieller Lungenerkankungen wurden im Zeitraum von 1995 bis 1999 Fragebögen von niedergelassenen Pneumologen und pneumologischen Krankenhausabteilungen ausgewertet. Insgesamt wurden in das vorliegende Register 1142 Patienten (579 männlich, 563 weiblich, Durchschnittsalter $51,1 \pm 15,3$ Jahre, Sarkoidose, $n=511$, exogen-allergische Alveolitis, $n=145$, idiopathischen Lungenfibrose $n=308$, Bronchiolitis obliterans organizing pneumonia (BOOP) $n=93$, andere $n=85$ ) aufgenommen. Bezogen auf das mittlere Durchschnittsalter trat die Sarkoidose in der 4. Dekade und die idiopathische Lungenfibrose in der 6. Dekade am häufigsten auf. Bei allen Erkrankungen stand zur weiterführenden Diagnostik im Vordergrund die Bronchoskopie mit bronchoalveolärer Lavage und transbronchialer Biopsie. Auffallend war in allen Krankheitsgruppen der noch geringe Einsatz der hochauflösenden Computertomographie des Thorax. Abgesehen von der Diagnosegruppe der BOOP lagen bei Frauen und Männern die Nichtraucheranteile deutlich über dem nach dem bundesdeutschen Durchschnitt errechneten Wert.

\section{Abstract}

From 1995 to 1999 we evaluated questionnaires sent by pulmonologists and departments of pulmonology in order to register interstitial lung diseases. On the whole 1142 patients ( 579 males, 563 females, mean age $51.1 \pm 15.3$ years, sarcoidosis, $n=511$, extrinsic allergic alveolitis, $\mathrm{n}=145$, idiopathic pulmonary fibrosis, $\mathrm{n}=308$, bronchiolitis obliterans organizing pneumonia (BOOP), $n=93$, others, $n=85$ ) were recorded in the registry. With reference to the mean age sarcoidosis occurred most frequently in the fourth decade and idiopathic pulmonary fibrosis in the sixth decade. In all these diseases bronchoscopy with bronchoalveolar lavage and transbronchial biopsy was predominantly used for further diagnosis. It was striking that high-resolution computed tomography of the thorax was still rarely used when diagnosing these diseases. Apart from the group with BOOP the number of non-smokers in men and women was decisively higher than the average of the population of Germany. der Humboldt-Universität zu Berlin (Charité), Cottbus ${ }^{2}$ Abt. Pneumologie/Allergologie, Ruhrlandklinik, Essen ${ }^{3}$ Krankenhaus Zehlendorf, Bereich Heckeshorn, Berlin ${ }^{4}$ Zentralklinikum Bad Berka GmbH, Bad Berka ${ }^{5}$ Fachkrankenhaus Coswig, Coswig ${ }^{6}$ Robert Koch Klinik, Leipzig 
Einleitung

Unter der Bezeichnung interstitielle Lungenerkrankung werden heute sehr heterogene Erkrankungen des Lungenparenchyms zusammengefasst, denen eine Beteiligung des Lungeninterstitiums am Krankheitsprozess gemeinsam ist. Große Probleme bereiten diese Erkrankungen durch ihre mannigfaltige und teilweise unverstandene Ätiologie bei der Diagnostik und Therapie [14].

Vor diesem Hintergrund gelang es in Deutschland 1995, gemeinsam mit der Deutschen Gesellschaft für Pneumologie sowie mit Drittmitteln das „Fibrose-Register“ in der Wissenschaftlichen Arbeitsgemeinschaft zur Therapie von Lungenkrankheiten (WATL) einzurichten. Es stellte erstmals für die gesamte Bundesrepublik eine Erfassungsmöglichkeit für Lungengerüsterkrankungen unterschiedlichster Genese dar. Das Ziel des Registers war es, Daten zur Patientenstruktur, Diagnostik, dem klinischen Bild und möglichen ätiopathogenetischen Faktoren zu erfassen und auszuwerten. Eine Zwischenauswertung unter der Berücksichtigung der 1995 gemeldeten Fälle ist bereits erfolgt [27,31]. Zwischenzeitlich haben auch andere Fachgesellschaften und Arbeitsgruppen Statements zur Diagnostik, Therapie und Erforschung von interstitiellen Lungenerkrankungen herausgegeben $[1,2,10]$.

\section{Material und Methode}

Zur Erfassung interstitieller Lungenerkrankungen wurden im Zeitraum von 1995 bis 1999 Fragebögen an niedergelassene Pneumologen und pneumologische Krankenhausabteilungen in Deutschland verschickt. Die Evaluation umfasst die anonymisierten Daten der Patienten (Geburtsdatum, Geschlecht und Initialen), die Diagnosen nach ICD 9 und das Datum der Erstdiagnose. Im Folgenden wurde die Diagnosefindung und -sicherung mittels invasiver Diagnostik (bronchoalveoläre Lavage, transbronchiale Biopsie, Thorakoskopie, Thorakotomie, Mediastinoskopie), durchgeführter radiologischer Maßnahmen (Röntgen des Thorax, Computertomographie, hochauflösende Computertomographie) und Funktionsuntersuchungen (Spirometrie, Blutgasanalyse in Ruhe und bei Belastung, Ganzkörperplethysmographie, CO-Transfer, EKG, Echokardiographie, Rechtsherzkatheter) abgefragt. Das klinische Erscheinungsbild der Erkrankung wurde durch die Erfassung weiterer Organbeteiligungen (Haut, Niere, Gelenke, Auge, Herz, ZNS, Leber, oberer Respirationstrakt, sonstige Organe) sowie der Beschwerden (Husten, Auswurf, Ruhedyspnoe, Belastungsdyspnoe, Fieber) und der klinischen Befunde (Knistern, Rechtsherzbelastung, Trommelschlegelfinger, Zyano- se, Löfgren-Syndrom) ermittelt. Es folgten Daten über das Rauchverhalten sowie über die Wohnverhältnisse und besondere Freizeitaktivitäten. Schließlich wurde nach dem Befund und dessen Risikofaktoren gefragt.

In der statistischen Auswertung der Daten wurde zunächst eine Alters- und Geschlechtsverteilungsprüfung auf Normal- bzw. Gleichverteilung mittels Kolmogorov-Smirnov- bzw. $\chi^{2}$-Test durchgeführt. Die Prüfung der Unterschiede bezüglich der Altersverteilung zwischen den Geschlechtern erfolgte mit dem U-Test nach Mann und Whitney bzw. dem t-Test. Signifikante Abweichungen zwischen den einzelnen Erkrankungsgruppen und den Geschlechtern bei Klinik und Beschwerden sowie den Organbeteiligungen wurden mit dem $\chi^{2}$-Test nach Pearson bzw. dem Fisher-Test berechnet. Zum Vergleich des Rauchverhaltens wurden Daten des statistischen Bundesamtes aus dem Jahr 1995 herangezogen und der $\chi^{2}$-Test zur Signifikanzprüfung etwaiger Differenzen benutzt. Für die Aufbereitung der Daten sowie deren statistischer Auswertung diente das Programm SPSS, Version 10.0.

\section{Ergebnisse}

Insgesamt wurden in das vorliegende Register 1142 Patienten aufgenommen (Tab. 1). Es handelte sich um 511 Patienten mit einer Sarkoidose (44,7\%), 145 mit exogen-allergischer Alveolitis (EAA) (12,7\%), 308 mit einer idiopathischen Lungenfibrose (IPF) (27,0\%), 93 mit einer Bronchiolitis obliterans organizing pneumonia (BOOP) $(8,1 \%)$ und $85(7,4 \%)$ mit anderen interstitiellen Krankheitsbildern, die nach ICD-9 eingruppiert wurden: 446,4 (M. Wegener, $n=5$ ), 501.0 (Asbestose, $n=19$ ), 502.0 (Pneumokoniose durch sonstige Kieselsäuren oder kieselsaure Salze, $n=11$ ), 503.0 (Pneumokoniose durch Einatmung von anderem anorganischem Staub, $n=6$ ), 507.1 (Pneumonien durch Eindringen von Öl und Essenzen durch die oberen Luftwege, $n=1$ ), 508.0 (akute pulmonale Manifestationen durch Bestrahlung, $n=5$ ), 516.0 (alveoläres Lungenproteinose-Syndrom, $n=10$ ), 516.1 (idiopathische pulmonale Hämosiderose, $n=7$ ), 517.0 (rheumatoide Lunge, $\mathrm{n}=14$ ), 517.1 (rheumatoide Pneumonie, $\mathrm{n}=1$ ), 517.2 (Lungenbeteiligung bei Systemsklerose, $n=6$ ).

\section{Altersstruktur}

Das Durchschnittsalter $( \pm$ SD) des Gesamtkollektivs lag bei 51,1 \pm 15,3 Jahren (Minimum 11, Maximum 88 Jahre) (Tab.1). Die Patienten mit Sarkoidose waren mit 43,5 Jahren deutlich jünger (Abb.1). Die Altersverteilung ergab bei der Sarkoidose bei den

Tab. 1 Altersstruktur des Gesamtkollektivs. Test auf Normalverteilung nach Kolmogorov-Smirnov

\begin{tabular}{|c|c|c|c|c|c|c|}
\hline Diagnosegruppen & $n$ & $\begin{array}{l}\bar{x} \text {-Alter } \\
\text { (Jahre) }\end{array}$ & $\begin{array}{l} \pm S D \\
\text { (Jahre) }\end{array}$ & $\begin{array}{l}\min \\
\text { (Jahre) }\end{array}$ & $\begin{array}{l}\max \\
\text { (Jahre) }\end{array}$ & $\begin{array}{l}\text { Kolmogorov-Smirnov } \\
p\end{array}$ \\
\hline Sarkoidose & 511 & 43,5 & 14,0 & 19 & 78 & $<0,001$ \\
\hline exogen-allergische Alveolitis & 145 & 52,6 & 13,5 & 11 & 81 & $=0,018$ \\
\hline idiopathische Lungenfibrose & 308 & 61,2 & 11,6 & 27 & 88 & $=0,115$ \\
\hline$B O O P$ & 93 & 52,4 & 15,6 & 22 & 81 & $=0,372$ \\
\hline sonstige & 85 & 55,6 & 13,5 & 16 & 88 & $=0,376$ \\
\hline insgesamt & 1142 & 51,1 & 15,3 & 11 & 88 & $<0,001$ \\
\hline
\end{tabular}




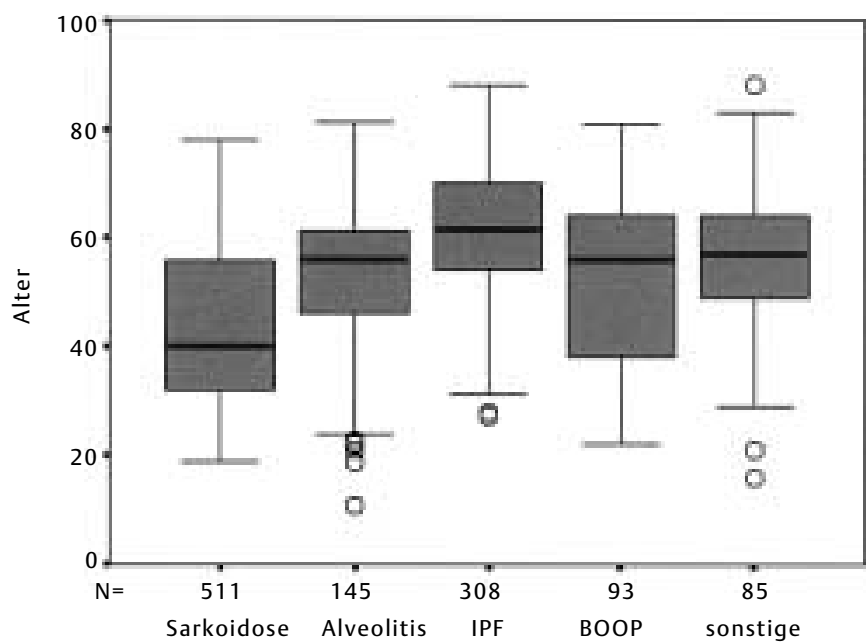

Abb. 1 Boxplot der Altersverteilung innerhalb der einzelnen Diagnosegruppen (Median, 25. und 75. Perzentil, Minimum, Maximum, 0 Ausreißer.

Männern eine Kumulierung vom 25. bis 35. Lebensjahr (Abb. 2). Bei den Frauen dagegen wurden zwei Altersgipfel zwischen dem 25. und 45. sowie dem 55. und 60. Lebensjahr beobachtet (Abb. 3).

Die Altersschichtungen der Gesamt- und der Teilkollektive zeigten signifikante Abweichungen von der Normalverteilung im Kolmogorov-Smirnov-Anpassungstest. Hiervon ausgenommen waren die IPF- und BOOP-Erkrankten sowie die Gruppe der sonstigen Fälle und die Untergruppe der männlichen EAA-Patienten, welche sich mit hinreichender Signifikanz als normalverteilt erwiesen (Tab. 1, 2 u. 3). Die Sarkoidosepatienten waren signifikant jünger und die IPF-Patienten signifikant älter als die Patienten in den übrigen Diagnosegruppen.

\section{Geschlechtsverteilung}

Die 1142 Patienten des Registers verteilen sich auf 579 Männer $(50,7 \%)$ und 563 Frauen $(49,3 \%)$ (Unterschied $\chi^{2}$-Test: n.s.) (Tab. 2 u. 3). In den Untergruppen der Sarkoidose- bzw. der BOOP-Fälle konnten ebenso keine signifikanten Unterschiede in der Geschlechtsverteilung bei 251 Männern vs. 260 Frauen bzw. 42 Männern vs. 51 Frauen ermittelt werden. Signifikante Unterschiede fanden sich jedoch bei EAA-Patienten mit 54 Männern und 91 Frauen $\left(\chi^{2}\right.$-Test: $\left.p<0,01\right)$, den IPF-Erkrankten mit 176 Männern und 132 Frauen $\left(\chi^{2}\right.$-Test: $\left.\mathrm{p}<0,05\right)$ und den sonstigen Fällen mit 56 Männern und 29 Frauen. Eine signifikante Abweichung der Altersschichtung zwischen den beiden Geschlechtern war nur bei den Sarkoidosepatienten mit Frauen in den höheren Altersgruppen festzustellen (Mann-Whitney-Test, $\mathrm{p}<0,001$ ). In den übrigen vier Krankheitsgruppen waren Männer und Frauen in den einzelnen Altersklassen demgemäß gleich häufig erkrankt.

\section{Klinik}

Im Gesamtkollektiv wurden als extrapulmonale Organbeteiligung insgesamt am häufigsten die Haut ( $\mathrm{n}=53$ Patienten) und die Gelenke ( $n=77$ Patienten) registriert. Es folgen mit größerem Abstand die Leber $(n=18)$, Augen $(n=14)$, oberer Respirationstrakt $(n=11)$, Herz $(n=10)$, Nieren $(n=5), Z N S(n=3)$ und sonsti-

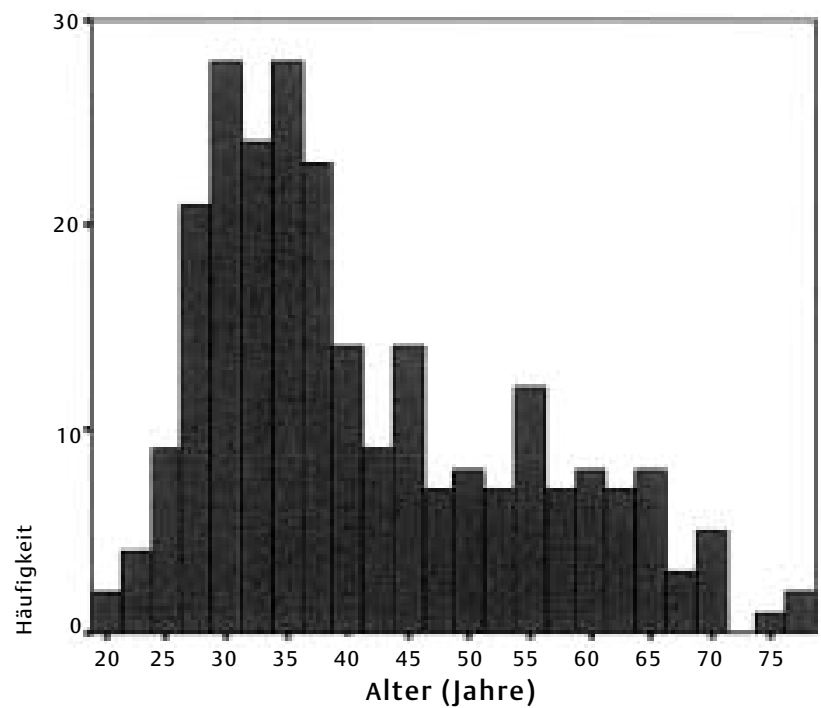

Abb. 2 Altersverteilung der Männer bei Sarkoidose $(n=251)$.

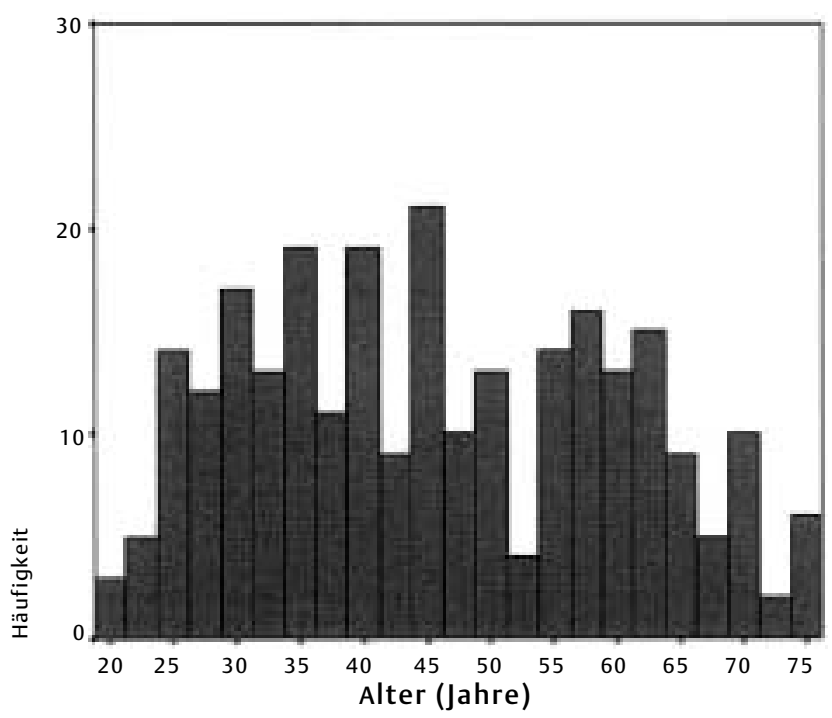

Abb. 3 Altersverteilung der Frauen bei Sarkoidose $(n=260)$.

ge Organe ( $n=45)$, hierunter war am häufigsten eine Manifestation an den lymphatischen Organen ( $\mathrm{n}=19)$.

Signifikant häufig fanden sich extrapulmonale Beteiligungen bei den Sarkoidoseerkrankten bezüglich der Haut $(n=44, p<0,001)$, Gelenke $(n=45, p<0,001)$, Augen $(n=13, p<0,01)$ und Leber $(n=16, p<0,01)$. In den übrigen Diagnosegruppen trat eine extrapulmonale Organbeteiligung in sehr geringer Anzahl auf. Eine signifikante Bevorzugung des weiblichen Geschlechts fand sich bezüglich der Gelenkbeteiligung bei IPF (Männer: 1 von 175; Frauen 7 von 125; $\mathrm{p}<0,05)$ und bei den sonstigen Erkrankungen (Männer: 3 von 53; Frauen 9 von 20; $p<0,01$ ).

Unter den Angaben zu Beschwerden und Befunden dominierten Husten ( $\mathrm{n}=709)$ und Belastungsdyspnoe $(\mathrm{n}=679)$, mit Abstand gefolgt von Knistern bei der Auskultation $(n=391)$, Auswurf $(n=280)$, Fieber $(n=146)$ und Ruhedyspnoe $(n=127)$. Eine 
Tab. 2 Altersstruktur der männlichen Patienten. Test auf Normalverteilung nach Kolmogorov-Smirnov

\begin{tabular}{lllllll}
\hline Diagnosegruppen & $\boldsymbol{n}$ & $\begin{array}{l}\text { x-Alter } \\
\text { (Jahre) }\end{array}$ & $\begin{array}{l} \pm \text { SD } \\
\text { (Jahre) }\end{array}$ & $\begin{array}{l}\text { min } \\
\text { (Jahre) }\end{array}$ & $\begin{array}{l}\text { max } \\
\text { (Jahre) }\end{array}$ & $\begin{array}{l}\text { Kolmogorov-Smirnov } \\
\boldsymbol{p}\end{array}$ \\
\hline Sarkoidose & 251 & 41,2 & 13,1 & 19 & 78 & $<0,001$ \\
\hline exogen-allergische Alveolitis & 54 & 52,2 & 13,1 & 26 & 81 & $=0,659$ \\
\hline idiopathische Lungenfibrose & 176 & 61,1 & 11,3 & 31 & 88 & $=0,264$ \\
\hline BOOP & 42 & 52,6 & 16,8 & 22 & 80 & $=0,544$ \\
\hline sonstige & 56 & 55,1 & 13,6 & 16 & 88 & $=0,739$ \\
\hline insgesamt & 579 & 50,4 & 15,5 & 16 & 88 & $<0,001$ \\
\hline
\end{tabular}

Tab. 3 Altersstruktur der weiblichen Patienten. Test auf Normalverteilung nach Kolmogorov-Smirnov

\begin{tabular}{lrlllll}
\hline Diagnosegruppen & $\boldsymbol{n}$ & $\begin{array}{l}\text { x-Alter } \\
\text { (Jahre) }\end{array}$ & $\begin{array}{l} \pm \text { SD } \\
\text { (Jahre) }\end{array}$ & $\begin{array}{l}\text { min } \\
\text { (Jahre) }\end{array}$ & $\begin{array}{l}\text { max } \\
\text { (Jahre) }\end{array}$ & $\begin{array}{l}\text { Kolmogorov-Smirnov } \\
\boldsymbol{p}\end{array}$ \\
\hline Sarkoidose & 260 & 45,8 & 14,4 & 19 & 76 & $<0,05$ \\
\hline exogen-allergische Alveolitis & 91 & 52,8 & 13,8 & 11 & 76 & $<0,05$ \\
idiopathische Lungenfibrose & 132 & 61,5 & 12,1 & 27 & 85 & $=0,378$ \\
BOOP & 51 & 52,3 & 14,7 & 23 & 81 & $=0,818$ \\
sonstige & 29 & 56,4 & 13,3 & 21 & 83 & $=0,832$ \\
insgesamt & 563 & 51,8 & 15,1 & 11 & 85 & $<0,001$ \\
\hline
\end{tabular}

Tab. 4 Klinische Symptome und Befunde

\begin{tabular}{|c|c|c|c|c|c|c|c|c|c|c|c|c|}
\hline & \multicolumn{2}{|c|}{ Sarkoidose } & \multicolumn{2}{|c|}{$\begin{array}{l}\text { exogen-allergische } \\
\text { Alveolitis }\end{array}$} & \multicolumn{2}{|c|}{$\begin{array}{l}\text { idiopathische } \\
\text { Lungenfibrose }\end{array}$} & \multicolumn{2}{|c|}{ BOOP } & \multicolumn{2}{|c|}{ sonstige } & \multicolumn{2}{|c|}{ gesamt } \\
\hline & $n$ & $\%$ & $n$ & $\%$ & $n$ & $\%$ & $n$ & $\%$ & $n$ & $\%$ & $n$ & $\%$ \\
\hline Husten & 240 & 47 & 112 & 77 & 222 & 72 & 76 & 82 & 59 & 69 & 709 & 69 \\
\hline Auswurf & 75 & 15 & 42 & 29 & 108 & 35 & 27 & 29 & 28 & 33 & 280 & 33 \\
\hline Ruhedyspnoe & 20 & 4 & 21 & 15 & 65 & 21 & 12 & 13 & 9 & 11 & 127 & 11 \\
\hline Belastungs-Dyspnoe & 198 & 39 & 114 & 79 & 242 & 79 & 66 & 71 & 59 & 69 & 679 & 69 \\
\hline Fieber & 57 & 11 & 29 & 20 & 25 & 8 & 25 & 27 & 10 & 12 & 146 & 12 \\
\hline auskultatorisches Knistern & 47 & 9 & 85 & 59 & 200 & 65 & 29 & 31 & 30 & 35 & 391 & 35 \\
\hline Rechtsherzbelastung & 9 & 2 & 12 & 8 & 41 & 13 & 3 & 3 & 4 & 5 & 69 & 5 \\
\hline Trommelschlegelfinger & 4 & 1 & 9 & 6 & 33 & 11 & 3 & 3 & 3 & 4 & 52 & 4 \\
\hline Zyanose & 11 & 2 & 16 & 11 & 53 & 17 & 9 & 10 & 4 & 5 & 93 & 5 \\
\hline
\end{tabular}

Rechtsherzbelastung ( $n=69)$, Trommelschlegelfinger $(n=52)$, Zyanose $(n=93)$ sowie das Löfgren-Syndrom $(n=53)$ waren vergleichsweise seltener (Tab. 4).

Auffällige Häufungen der genannten Symptome und Befunde innerhalb einzelner Diagnosegruppen waren feststellbar (Tab. 4 u. 5). So kam Fieber bei EAA ( $n=29$; erwartet $n=18,5)$ und BOOP $(n=25$; erwartet 11,9$)$ signifikant häufiger vor. Knistern war signifikant seltener bei Sarkoidose ( $n=47$, erwartet 175) und signifikant häufiger bei IPF ( $n=200$, erwartet 105,5). Eine Rechtsherzbelastung ( $n=41$, erwartet 18,6$)$, Trommelschlegelfinger $(n=33$, erwartet 14,0$)$ Zyanose ( $n=53$, erwartet 25,1$)$ und Ruhedyspnoe
( $n=65$, erwartet 34,3 ) traten ebenfalls bei IPF gehäuft auf. Ein Löfgren-Syndrom wurde bei 52 Sarkoidosepatienten registriert. Sarkoidosepatienten litten signifikant seltener an Husten ( $n=240$, erwartet 317,2$)$, Auswurf $(n=75$, erwartet 125,3$)$ und Belastungsdyspnoe $(n=198$, erwartet 303,8$)$.

\section{Diagnostische Maßnahmen}

Bei den angewandten Funktionsuntersuchungen führten die Bodyplethysmographie $(94,1 \%)$ und die Blutgasanalyse unter Ruhebedingungen (92,8\%), gefolgt vom EKG (88,0\%) und der COTransfer-Messung (64,0\%). Eine einfache Spirometrie kam in 48,5\% der Fälle zum Einsatz; eine Echokardiographie wurde in 
Tab. 5 Klinische Symptome und Befunde der Patienten mit Sarkoidose, unterteilt nach den radiologischen Stadien

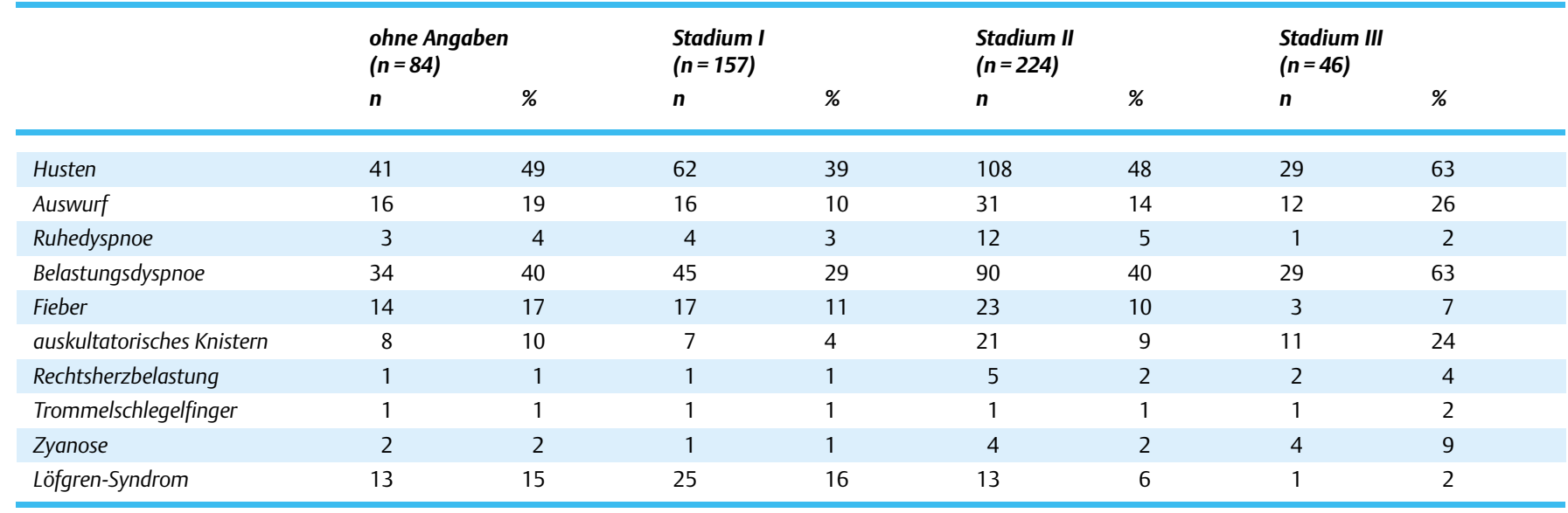

Tab. 6 Durchgeführte Funktionsdiagnostik

\begin{tabular}{|c|c|c|c|c|c|c|c|c|c|c|c|c|}
\hline & \multicolumn{2}{|c|}{ Sarkoidose } & \multicolumn{2}{|c|}{$\begin{array}{l}\text { exogen-allergische } \\
\text { Alveolitis }\end{array}$} & \multicolumn{2}{|c|}{$\begin{array}{l}\text { idiopathische } \\
\text { Lungenfibrose }\end{array}$} & \multicolumn{2}{|c|}{ BOOP } & \multicolumn{2}{|c|}{ sonstige } & \multicolumn{2}{|c|}{ gesamt } \\
\hline & $n$ & $\%$ & $n$ & $\%$ & $n$ & $\%$ & $n$ & $\%$ & $n$ & $\%$ & $n$ & $\%$ \\
\hline Spirometrie & 261 & 51,1 & 52 & 35,9 & 158 & 51,3 & 46 & 49,5 & 37 & 43,5 & 554 & 48,5 \\
\hline BGA in Ruhe & 475 & 93,0 & 131 & 90,3 & 288 & 93,5 & 87 & 93,5 & 79 & 92,9 & 1060 & 92,8 \\
\hline BGA bei Belastung & 389 & 76,1 & 117 & 80,7 & 216 & 70,1 & 59 & 63,4 & 54 & 63,5 & 835 & 73,1 \\
\hline Bodyplethysmographie & 483 & 94,5 & 141 & 97,2 & 284 & 92,2 & 86 & 92,5 & 81 & 95,3 & 1075 & 94,1 \\
\hline CO-Transfer & 319 & 62,4 & 98 & 67,6 & 228 & 74,0 & 39 & 41,9 & 47 & 55,3 & 731 & 64,0 \\
\hline EKG & 453 & 88,6 & 127 & 87,6 & 274 & 89,0 & 80 & 86,0 & 71 & 83,5 & 1005 & 88,0 \\
\hline Echokardiographie & 97 & 19,0 & 29 & 20,0 & 101 & 32,8 & 22 & 23,7 & 25 & 29,4 & 274 & 24,0 \\
\hline Rechtsherzkatheter & 25 & 4,9 & 21 & 14,5 & 46 & 14,9 & 6 & 6,5 & 7 & 8,2 & 105 & 9,2 \\
\hline
\end{tabular}

24,0\% und eine Rechtsherzkatheter-Untersuchung in nur 9,2\% der Fälle angewandt (Tab. 6 ).

Bei den radiologischen Verfahren war die konventionelle Röntgenaufnahme der Thoraxorgane die mit Abstand häufigste diagnostische Maßnahme (96,8\%). Eine Computertomographie wurde in $69,9 \%$ der Fälle vorgenommen, davon in $26,3 \%$ ein HR-CT und in $43,6 \%$ eine konventionelle Computertomographie. Bei Sarkoidose- und BOOP-Patienten lag die Häufigkeit des HR-CT bei 17,6 bzw. 21,5\% und damit gering unter dem Durchschnitt (Tab. 7).

Im Rahmen der invasiven Diagnostik wurden in 85,8\% der Fälle bronchoalveoläre Lavagen und in 69,9\% transbronchiale Biopsien durchgeführt, was sich in einer ähnlichen prozentualen Verteilung auch innerhalb der einzelnen Diagnosegruppen zeigte. Im Vergleich zu 2 anderen Studien war der Anteil der transbronchialen Biopsien mehr als doppelt so hoch [31]. Thorakoskopien und Thorakotomien wurden insgesamt sehr viel seltener angewandt; lediglich bei den BOOP war die Thorakotomie zu Diagnosezwecken in 25,8\% der Erkrankten erforderlich. Eine Mediastinoskopie erfolgte mit zwei Ausnahmen nur bei Sarkoidosepatienten in 9,6\% der Fälle (Tab. 8).
Bei den vorliegenden Angaben zur Diagnosesicherung ist zu beachten, dass nur ein Verfahren genannt werden sollte, das zur Diagnose führte. In insgesamt $48,0 \%$ der Fälle wurde eine transbronchiale Biopsie und in 24,8\% eine bronchoalveoläre Lavage angegeben. In 15,7\% der Fälle wurde die Diagnose klinisch-radiologisch gestellt. Bei den Sarkoidosepatienten war eine transbronchiale Biopsie in überdurchschnittlichem Maße (59,1\%) diagnostisch. Die Mediastinoskopie erbrachte in fast allen Fälle (45 von 49; 91,8\%) die Diagnose. Mittels bronchoalveolärer Lavage ließen sich überdurchschnittlich häufig (37,2\%) die Diagnose einer exogen-allergischen Alveolitis stellen. Innerhalb der IPF- und BOOPKrankheitsgruppen konnte in 7,1 bzw. 22,6\% die Diagnose erst durch eine Thorakotomie ermittelt werden; anderseits wurde hier die Diagnose in 26,0 bzw. 21,5\% der Fälle bereits klinisch-radiologisch gestellt (Tab. 9 ).

\section{Rauchverhalten}

Die 1142 Patienten des Gesamtregisters lassen sich nach ihrem Rauchverhalten in folgende drei Gruppen einteilen: 201 Raucher, 303 ehemalige Raucher und 606 Nieraucher. Bei 32 Patienten wurden diesbezüglich keine Angaben gemacht. Die Verteilung innerhalb der einzelnen Diagnosegruppen ist Abb. 4 zu entnehmen. Zum statistischen Vergleich erfolgte die Aufteilung der Er- 
Tab. 7 Durchgeführte radiologische Diagnostik

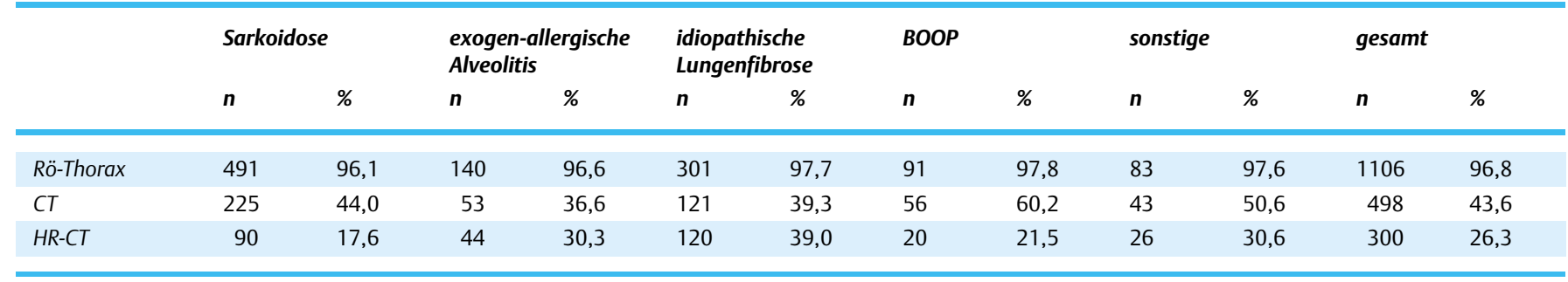

Tab. 8 Durchgeführte invasive Diagnostik

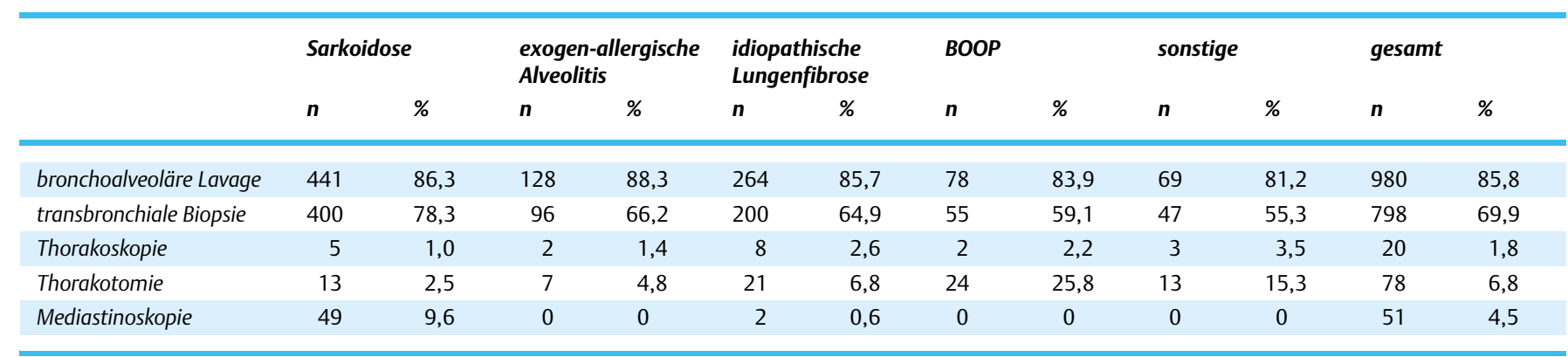

Tab. 9 Verfahren zur Diagnosesicherung

\begin{tabular}{|c|c|c|c|c|c|c|c|c|c|c|c|c|}
\hline & \multicolumn{2}{|c|}{ Sarkoidose } & \multicolumn{2}{|c|}{$\begin{array}{l}\text { exogen-allergische } \\
\text { Alveolitis }\end{array}$} & \multicolumn{2}{|c|}{$\begin{array}{l}\text { idiopathische } \\
\text { Lungenfibrose }\end{array}$} & \multicolumn{2}{|c|}{ BOOP } & \multicolumn{2}{|c|}{ sonstige } & \multicolumn{2}{|c|}{ gesamt } \\
\hline & $n$ & $\%$ & $n$ & $\%$ & $n$ & $\%$ & $n$ & $\%$ & $n$ & $\%$ & $n$ & $\%$ \\
\hline klinisch-radiolog. Diagnostik & 36 & 7,0 & 17 & 11,7 & 80 & 26,0 & 20 & 21,5 & 26 & 30,6 & 179 & 15,7 \\
\hline bronchoalveoläre Lavage & 112 & 21,9 & 54 & 37,2 & 71 & 23,1 & 22 & 23,7 & 24 & 28,2 & 283 & 24,8 \\
\hline transbronchiale Biopsie & 302 & 59,1 & 68 & 46,9 & 128 & 41,6 & 28 & 30,1 & 22 & 25,9 & 548 & 48,0 \\
\hline Mediastinoskopie & 45 & 8,8 & 0 & 0 & 0 & 0 & 0 & 0 & 0 & 0 & 45 & 3,9 \\
\hline
\end{tabular}

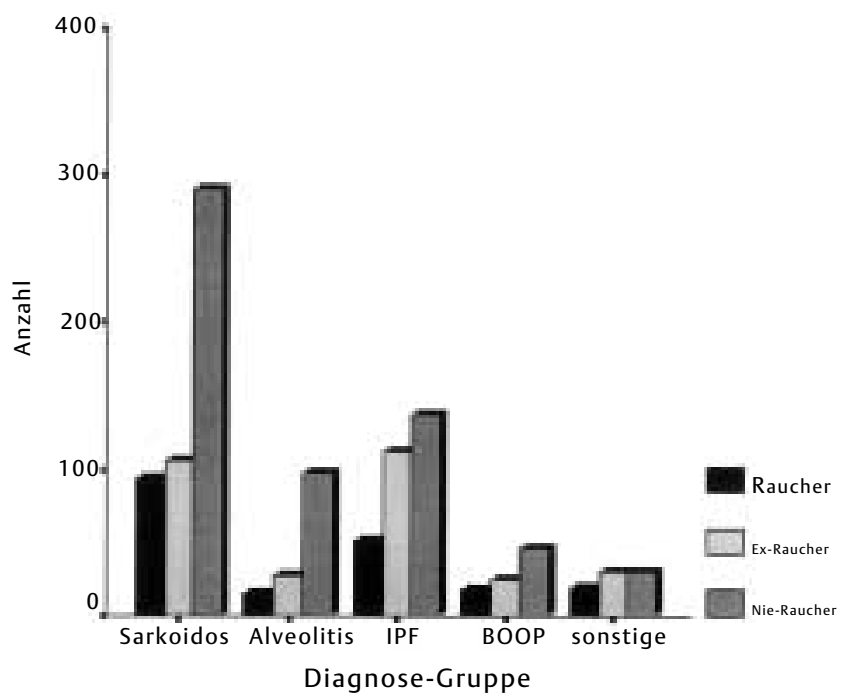

Abb. 4 Rauchverhalten einzelner Diagnosegruppen $(n=1110)$. Bei 32 Patienten fehlte die Raucheranamnese. krankten in Raucher und Nichtraucher, wobei letzterer Gruppe die ehemaligen Raucher zugeschlagen wurden. Abgesehen von der Diagnosegruppe der BOOP- und sonstigen Patienten lagen bei Frauen und Männern die Nichtraucheranteile deutlich über dem nach dem bundesdeutschen Durchschnitt errechneten Wert. Die detaillierten Daten sind getrennt nach Geschlechtern in Tab. 10 u. 11 aufgeführt.

\section{Diskussion}

Die abschließende Auswertung des Registers der Meldungen über den Zeitraum von 1995 bis 1999 erfolgte bei einem Stand von 1142 Patienten. Die Sarkoidose war mit Abstand die häufigste interstitielle Lungenerkrankung. Die Hälfte der Fälle des Registers wurde von Sarkoidosepatienten eingenommen, mehr als ein Drittel entfiel auf die idiopathische Lungenfibrose, die übrigen Erkrankten verteilten sich zu jeweils ungefähr gleichen Teilen auf Bronchiolitis obliterans organizing pneumonia, exogen-aller- 
Tab. 10 Rauchverhalten der männlichen Patienten nach Diagnosegruppen im Vergleich mit dem Bundesdurchschnitt $\left(\chi^{2}\right.$-Test)

\begin{tabular}{|c|c|c|c|c|c|}
\hline \multirow[t]{2}{*}{ Diagnose } & \multicolumn{2}{|c|}{ Raucher } & \multicolumn{2}{|c|}{ Nichtraucher } & \multirow{2}{*}{$\begin{array}{l}\text { Abweichung } \\
\text { vom Bundes- } \\
\text { schnitt (35,6\%) } \\
\text { p }\end{array}$} \\
\hline & $n$ & $\%$ & $n$ & $\%$ & \\
\hline Sarkoidose & 58 & 24,2 & 182 & 75,8 & $<0,001$ \\
\hline Alveolitis & 10 & 18,5 & 44 & 81,5 & $<0,01$ \\
\hline $\begin{array}{l}\text { idiopathische } \\
\text { Lungenfibrose }\end{array}$ & 41 & 23,8 & 131 & 76,2 & $<0,01$ \\
\hline$B O O P$ & 10 & 24,4 & 31 & 75,6 & $>0,05$ (n. s.) \\
\hline sonstige & 17 & 30,4 & 39 & 69,6 & $>0,05$ (n. s.) \\
\hline gesamt & 136 & 24,2 & 427 & 75,8 & $<0,001$ \\
\hline
\end{tabular}

Tab. 11 Rauchverhalten der weiblichen Patienten nach Diagnosegruppen im Vergleich mit dem Bundesdurchschnitt $\left(\chi^{2}\right.$-Test)

\begin{tabular}{|c|c|c|c|c|c|}
\hline \multirow[t]{2}{*}{ Diagnose } & \multicolumn{2}{|c|}{ Raucher } & \multicolumn{2}{|c|}{ Nichtraucher } & \multirow{2}{*}{$\begin{array}{l}\text { Abweichung } \\
\text { vom Bundes- } \\
\text { schnitt (21,5\%) } \\
p\end{array}$} \\
\hline & $n$ & $\%$ & $n$ & $\%$ & \\
\hline Sarkoidose & 36 & 14,3 & 216 & 85,7 & $<0,01$ \\
\hline Alveolitis & 6 & 6,8 & 82 & 93,2 & $<0,001$ \\
\hline $\begin{array}{l}\text { idiopathische } \\
\text { Lungenfibrose }\end{array}$ & 11 & 8,4 & 120 & 91,6 & $<0,001$ \\
\hline$B O O P$ & 9 & 18,0 & 41 & 82,0 & $>0,05$ (n.s.) \\
\hline sonstige & 3 & 11,5 & 23 & 88,5 & $>0,05$ (n.s.) \\
\hline gesamt & 65 & 11,9 & 482 & 88,1 & $<0,001$ \\
\hline
\end{tabular}

gische Alveolitis sowie die sonstigen Fälle. Rückschlüsse auf Inzidenz und Prävalenz dieser Erkrankungen in der Bevölkerung Deutschlands lassen sich nicht ziehen. Die Verteilung bzw. das Verhältnis der jeweiligen Häufigkeiten stimmt mit bisher publizierten Daten überein. Hierbei fallen insbesondere das regional sehr unterschiedliche Prävalenzmuster der Sarkoidose sowie die regionalen Unterschiede in der Allergenexposition im Falle der exogen-allergischen Alveolitis ins Gewicht [8,12,23,24,29].

In Hinsicht auf eine Geschlechtsbevorzugung fanden sich signifikante Unterschiede nur bei der exogen-allergischen Alveolitis (häufiger Frauen) und der idiopathischen Lungenfibrose (häufiger Männer). Der Trend zur Überrepräsentation der Männer innerhalb der IPF-Gruppe wurde schon in vergangenen Studien aufgezeigt $[8,9,28,30,32]$. Ihr biologischer Hintergrund ist jedoch unklar. Bei der Sarkoidose fanden wir keine Geschlechtsbevorzugung. Insbesondere bei der Sarkoidose ist die Geschlechtsverteilung abhängig vom Erkrankungsalter und wird nicht unerheblich durch die angewandte Diagnostik (z. B. eindeutige Klinik und Thorax-Röntgen) beeinflusst [15,16].

Die Analyse des Alters zum Zeitpunkt der Diagnose macht deutlich, dass bei einem durchschnittlichen Erkrankungsalter von 52
Jahren innerhalb des gesamten Registers, IPF-Patienten davon abweichend 10 Jahre älter und Sarkoidosepatienten 5 Jahre jünger sind, wenn sie erkranken. In einer früheren Studie war das Durchschnittsalter der Sarkoidosepatienten um 10 Jahre jünger [22]. Für die Sarkoidose ist bei der auffällig inhomogenen Verteilung des Alters allerdings die Angabe eines mittleren Erkrankungsalters besonders kritisch zu bewerten. In der Gruppe der IPF-Patienten liegt das Erkrankungsalter in der gleichen Größenordnung wie in anderen Arbeiten angegeben $[5,17]$.

Während das Erkrankungsalter der IPF-Patienten einer Normalverteilung folgt, lässt sich dies für die Sarkoidose nicht bestätigen. Bei Frauen findet sich eine zweigipflige Verteilung mit einem zweiten Altersgipfel im 55. - 60. Lebensjahr, während Männer mit einem Altersgipfel zwischen dem 25. und 35. Lebensjahr erkranken und danach bei einer vergleichsweise niedrigen Erkrankungshäufigkeit bleiben. Die Altersverteilung der Männer, aber nicht der Frauen, entspricht einer früheren Studie aus der ehemaligen DDR [25]. Schon in der Vergangenheit wurde vor allem für die Frauengruppe ein hormoneller Einfluss auf die Erkrankung verantwortlich gemacht, da hier erhöhte Inzidenzen während des frühen Erwachsenenalters und danach erst wieder peri- und postmenopausal erreicht werden $[15,16,33]$.

Bei der exogen-allergischen Alveolitis zeigen sich ebenfalls augenfällige Häufigkeitsunterschiede zwischen den Geschlechtern. Während Männer im Mittel mit 52 Jahren erkranken - hier liegt eine Normalverteilung vor -, weisen Frauen einen deutlichen Erkrankungsgipfel nach der Menopause auf und haben davor ein vergleichsweise geringeres Erkrankungsrisiko. Rückschlüsse auf einen geschlechtsspezifischen Faktor erscheinen aber bei dieser Erkrankung nur bedingt zulässig, da zweifelsohne der Zeitpunkt der Antigenexposition als Vorbedingung für den Ausbruch der Immunreaktion die Hauptrolle spielen wird (z. B. Tierhaltung im höheren Lebensalter). Potenzielle genetische Prädisposition könnte darüber hinaus eines der Geschlechter bevorzugen.

Zur Diagnosestellung der interstitiellen Lungenerkrankungen wurde am häufigsten eine BAL durchgeführt und in vielen Fällen die Gewinnung einer Histologie angestrebt. Dabei ist zu beachten, dass in den Fragebögen nur ein Verfahren genannt werden sollte, das zur Diagnose führte. Zurückblickend ist hier anzumerken, dass unter Umständen eine Mehrfachnennung der angewendeten Verfahren günstiger gewesen wäre, da die Diagnose oft aus mehreren Befunden gestellt wurde.

In den Gruppen der an Sarkoidose und an einer exogen-allergischen Alveolitis Erkrankten konnten durch BAL und TBB in über 86\% der Fälle die Diagnose gestellt werden. Die Bronchoskopie stellte somit für diese beiden Erkrankungen das wichtigste diagnostische Einsatzmittel dar und ermöglichte es, auf invasive transthorakale Eingriffe zu verzichten. Bei BOOP- und IPF-Patienten erreichte die transbronchial gewonnene Histologie eine Trefferquote von knapp $42 \%$. Hier ist kritisch anzumerken, dass nach heutiger Auffassung die IFP nicht durch eine TBB gesichert bzw. diagnostiziert werden kann, da diese Biopsieform für die Beurteilung der Verteilung der histopathologischen Läsion innerhalb des sekundären Lobulus zu klein ist. Eine pathologische Klassifizierung in UIP, DIP, RBILD, AIP oder NSIP setzt eine chirurgische Biopsie voraus $[4,17,18]$. 
Unter den radiologischen Maßnahmen wurde neben der routinemäßig angefertigten Röntgen-Thorax-Aufnahme in weniger als der Hälfte der Fälle eine Computertomographie und in weniger als einem Viertel der Patienten eine High-Resolution-Computertomographie (HR-CT) vorgenommen. Hier muss noch Aufklärungsarbeit geleistet werden, da bei interstitiellen Lungenerkrankungen die Computertomographie immer auch in HR-Technik durchzuführen ist, wobei insbesonders bei der idiopathischen Lungenfibrose zwischen dem HR-CT-Befund und der Überlebensrate eine signifikante Beziehung besteht [11]. Die fehlenden Angaben für die Röntgenuntersuchungen des Thorax in $3,2 \%$ der Fälle ist möglicherweise so zu werten, dass der diagnostizierenden und meldenden Einrichtung bereits Fremdaufnahmen vorlagen. Eine Ausnahme bildete bei den angewandten radiologischen Verfahren die BOOP, bei der aufgrund der eher unspezifischen Veränderungen der Röntgen-Thorax-Aufnahme häufiger eine Computertomographie vorgenommen wurde.

Im Rahmen der Funktionsdiagnostik gehörten die Blutgasanalysen sowie die Bodyplethysmographie zum „Standardprogramm“, das jeweils in über $90 \%$ der Fälle durchgeführt wurde.

Bei den klinischen Befunden waren die Schwerpunkte des vorliegenden Registers einerseits auf die Erfassung von extrapulmonalen Organbeteiligungen und andererseits auf die typischen respiratorischen Beschwerden und deren Folgeerscheinungen gerichtet. Die Erkrankung mit den häufigsten extrapulmonalen Befunden stellte die Gruppe der Sarkoidose dar, was sich mit ihrem Charakter als Multisystemerkrankung vereinbaren lässt. Die Haut war in Form des Erythema nodosum und die Gelenke durch eine Arthritis oder Arthralgie am häufigsten mitbeteiligt, gefolgt von Leber und Auge. In früheren Studien wurden deutlich höhere Prozentsätze für die jeweiligen extrapulmonalen Organbeteiligungen angegeben als in der vorliegenden Arbeit [20,21]. Dies legt nahe, dass im vorliegenden Register nicht systematisch nach anderen Manifestationen gesucht wurde (z.B. keine regelmäßige Leberbiopsie oder augenärztliche Untersuchung), oder dass Patienten mit führenden extrapulmonalen Befunden nicht immer auch einem Pneumologen vorgestellt wurden. Im gesamten Register erwiesen sich die Gelenke als die am häufigsten beteiligten Organe.

Klinisch dominierte an pulmonalen Beschwerden der Husten. Er korrelierte in nahezu allen Fällen in gleicher Häufigkeit mit der Belastungsdyspnoe. Während für die Sarkoidose seltener Beschwerden angegeben wurden, konnte bei der exogen-allergischen Alveolitis und der BOOP öfters ein Knistern auskultiert werden. Unter den IPF-Patienten konnten häufiger Symptome der chronischen Einschränkung der respiratorischen und konsekutiv der kardialen Funktion beobachtet werden. Der Anteil der Patienten mit Rechtsherzbelastung, Zyanose und Trommelschlegelfingern war deutlich erhöht, in geringerem Umfang war dies auch bei der exogen-allergischen Alveolitis der Fall. Der Vergleich der unterschiedlichen Sarkoidosestadien erbrachte eine Verschiebung der Symptome und Befunde von Fieber im Stadium I zu den Anzeichen des chronischen Verlaufes und der Fibrose im Stadium III mit Belastungsdyspnoe, Husten und auskultatorischem Knistern.
Für einen direkten Zusammenhang zwischen den Krankheitsgruppen und dem Rauchverhalten konnte in der vorliegenden Arbeit kein Anhalt gefunden werden. Wie auch frühere Studien belegen, wurde das inhalative Zigarettenrauchen - im Gegensatz zur Histiozytose $X[6,26]$ und zur desquamativen interstitiellen Pneumonie [13] - offenbar nicht als ein krankheitsdeterminierender Hauptfaktor im Geschehen der hier analysierten interstitiellen Lungenkrankheiten verifiziert. Auffällig erscheint hierbei lediglich der signifikant höhere Nichtraucheranteil unter den Frauen, was sich mit den epidemiologischen Daten der „gesunden" rauchenden Normalbevölkerung deckt. Der erhöhte Anteil der ehemaligen Raucher unter den IPF-Patienten ließe sich zum einen mit einem verstärkten Einstellen des Zigarettenkonsums bei zunehmender Dyspnoe erklären, war jedoch an anderer Stelle schon als möglicher relevanter ätiologischer Faktor gesehen worden [3,7], wobei allerdings keine Beziehung zur Überlebensrate gefunden wurde [19].

\section{Schlussfolgerung}

In dieser über 5 Jahre dauernden Studie wurde nachgewiesen, dass die Sarkoidose die häufigste interstitielle Erkrankung ist, gefolgt von der idiopathischen Lungenfibrose, der exogen-allergischen Alveolitis und der BOOP. Bezogen auf das mittlere Durchschnittsalter trat die Sarkoidose in der 4. Dekade und die idiopathische Lungenfibrose in der 6. Dekade am häufigsten auf. Die exogen-allergische Alveolitis und die BOOP lagen altersgemäß dazwischen. Bei allen Erkrankungen stand zur weiterführenden Diagnostik im Vordergrund die Bronchoskopie mit bronchoalveolärer Lavage und transbronchialer Biopsie. Überraschend war in allen Krankheitsgruppen der noch geringe Einsatz der hochauflösenden Computertomographie des Thorax. Im Gegensatz zu anderen Lungenerkrankungen wie Lungenkarzinom oder COPD lag der Anteil der Raucher niedriger. 
Prof. Dr. med. Hans Schweisfurth, Dr. med. Ch. Kieslich, U. Gereke III. Medizinische Klinik - Pneumologie

Carl-Thiem-Klinikum

Akademisches Lehrkrankenhaus der Humboldt-Universität zu Berlin (Charité)

Thiemstr. 111

03048 Cottbus

Prof. Dr. med. U. Costabel, Dr. med. N. Satake

Abteilung Pneumologie/Allergologie

Ruhrlandklinik

Tüschener Weg 40

45239 Essen

Prof. Dr. med. R. Loddenkemper, Dr. med. N. Schönfeld

Krankenhaus Zehlendorf

Bereich Heckeshorn

Zum Heckeshorn 33

14109 Berlin

Dr. med. I. Mäder, Dr. med. D. Treutler

Zentralklinikum Bad Berka GmbH

Robert-Koch-Allee 9

99437 Bad Berka

Priv.-Doz. Dr. med. W. Matthiessen, Dr. med. C. Schmidt Fachkrankenhaus Coswig

Neucoswiger Str. 21

01610 Coswig

Prof. Dr. med. P. Leonhardt

Robert-Koch-Klinik

N.-Rumjanzow-Str. 100

04157 Leipzig

Prof. Dr. med. G. Siemon

Klinik für Erkrankungen der Atmungsorgane

Ludwigstr. 68

93093 Donaustauf

Dr. med. N. de Wall

Nordwest-Krankenhaus Sanderbusch

26454 Sande

\section{Weitere Mitautoren}

Dr. med. W. Frank

Johanniter-Krankenhaus

14929 Treuenbritzen

Dr. med. T. Gebhardt

Scheffelstr. 33

60318 Frankfurt/Main

Dr. med. S. Hummel

Fachkrankenhaus für Lungenkrankheiten und Thoraxchirurgie Karower Str. 11

13125 Berlin-Buch
Dr. med. R. Kropp

Bahnhofstr. 12

36037 Fulda

Dr. med. W. Mall

Städtisches Krankenhaus Heidehaus

Am Leineufer 70

30419 Hannover

Dr. med. V. Rausch

Evangelische und Johanniter Krankenanstalten

Steinbrinkstr. 96a

46145 Oberhausen

Prof. Dr. med. V. Schulz

Thoraxklinik der LVA

Amalienstr. 5

69126 Heidelberg

Prof. Dr. med. G. W. Sybrecht

Medizinische Klinik Universitätskliniken des Saarlandes

66421 Homburg/Saar

\section{Literatur}

${ }^{1}$ American Thoracic Society. Idiopathic pulmonary fibrosis: Diagnosis and treatment. International consensus statement. Am J Respir Crit Care Med 2000; 161: 646-664

2 American Thoracic Society. European Respiratory Society. International multidisciplinary consensus classification of the idiopathic interstitial pneumonias. Am J Respir Crit Care Med 2002; 165: 277-304

${ }^{3}$ Baumgartner KB, Samet JM, Stidley CA et al. Cigarette smoking: a risk factor for idiopathic pulmonary fibrosis. Am J Respir Crit Care Med 1997; 155: 242-248

${ }^{4}$ Behr J. Diagnostik der idiopathischen Lungenfibrose. Pneumologie 2001; 55: $144-148$

${ }^{5}$ Bjoraker JA, Ryu JH, Edwin MK et al. Prognostic significance of histopathologic subsets in idiopathic pulmonary fibrosis. Am J Respir Crit Care Med 1998; 157: 199-203

${ }^{6}$ Callebaut W, Demedts M, Verleden G. Pulmonary Langerhans' cell granulomatosis (histiocytosis X) clinical analysis of 8 cases. Acta Clin Belg 1998; 53: 337-343

${ }^{7}$ Carrington CB, Gaensler EA, Coutu RE et al. Natural history and treated course of usual and desquamative interstitial pneumonia. $\mathrm{N}$ Engl J Med 1978; 298: $801-809$

${ }^{8}$ Coultas DB, Zumwalt RE, Black WC et al. The epidemiology of interstitial lund diseases. Am J Respir Crit Care Med 1994; 150: 967- 972

${ }^{9}$ Crystal RG, Fulmer DJ, Roberts WC et al. Idiopathic pulmonary fibrosis: clinical, histologic, radiographic, physiologic, scintigraphic, cytologic, and biochemical aspects. Ann Intern Med 1976; 85: 769- 788

${ }^{10}$ Crystal RG et al. NHBLI workshop summery. Future research directions in idiopathic pulmonary fibrosis. Am J Respir Crit Care Med 2002; 166: $236-246$

${ }^{11}$ Gay SE et al. Idiopathic pulmonary fibrosis. Predicting response to therapy and survival. Am J Respir Crit Care Med 1998; 157: 1063 - 1072

12 Gideon NM, Mannion DM. Sarcoidosis mortality in the United States, 1979-1981: an analysis of multiple-cause mortality data. Am J Med 1996; 100: $423-427$

${ }^{13}$ Goeckenjan G. Respiratorische Bronchiolitis mit interstitieller Lungenerkrankung (RB-ILD). Pneumologie 2003; 57: 278-287

${ }^{14}$ Green FHY. Overview of pulmonary fibrosis. Chest 2002; 122: $334-339$

${ }^{15}$ Hillerdal G, Nou E, Osterman K et al. Sarcoidosis: Epidemiology and prognosis. A 15-year European study. Am Rev Respir Dis 1984; 130: $29-32$

${ }^{16}$ Hosoda Y, Yamaguchi M, Hiraga Y. Global epidemiology of sarcoidosis. Sarcoidosis 1997; 18: $681-694$ 
${ }^{17}$ Katzenstein AL, Myers JL. Idiopathic pulmonary fibrosis: clinical relevance of pathologic classification. Am J Respir Crit Care Med 1994; 150: $77-96$

${ }^{18}$ Katzenstein AL, Myer JL. State of art. Idiopathic pulmonary fibrosis. Clinical relevance of pathologic classification. Am J Respir Crit Care Med 1986; 157: $1301-1315$

${ }^{19}$ King TE et al. Idiopathic pulmonary fibrosis. Relationship between histopathologic features and mortality. Am J Respir Crit Care Med 2001; 164: $1025-1032$

${ }^{20}$ Kirsten D. Sarkoidose in Deutschland. Pneumologie 1995; 49: $378-382$

${ }^{21}$ Kirsten D, Maganussen H. Die Diagnostik und Differentialdiagnostik der Lungensarkoidose. In: Sarkoidose Up-Date 2000 Norderstedt: BoD GmbH, 2001

${ }^{22}$ Loddenkemper R, Kloppenborg A, Schoenfeld $\mathrm{N}$ et al. Clinical findings in 715 patients with newly detected pulmonary sarcoidosis - results of a cooperative study in former West Germany and Switzerland. Sarcoidosis Vasc Diffuse Lung Dis 1998; 15: 178-182

${ }^{23}$ Marx JJ et al. Cohort studies of immunologic lung disease among Wisconsin dairy farmers. Am J Int Med 1990; 18: 263

${ }^{24}$ Reynolds HY. Diagnostic and management strategies of diffuse interstitial lung disease. Chest 1998; 113: $192-202$

${ }^{25}$ Scharkoff T. Epidemiologie der Sarkoidose. Pneumologie 1993; 47: $588-592$
${ }^{26}$ Schönfeld N. Pulmonale Langerhans-Zell-Histiocytosis. Pneumologie 2003; 57: 159-165

${ }^{27}$ Schweisfurth H, Costabel U, Kropp R et al. Mitteilung der Wissenschaftlichen Arbeitsgemeinschaft für die Therapie von Lungenkrankheiten (WATL): Deutsches Fibroseregister mit ersten Ergebnissen. Pneumologie 1996; 50: 899-901

${ }^{28}$ Scott J, Johnston I, Britton J. What causes cryptogenic fibrosing alveolitis? A case-control study of environmental exposure to dust. British Med J 1990; 301: 1015- 1017

${ }^{29}$ Sharma OP, Badr A. Sarcoidosis: diagnosis, staging, a newer diagnostic modalities. Clin Pulm Med 1994; 1: 223 -231

${ }^{30}$ Stack BH, Choo-Kang YF, Heard BE. The prognosis of cryptogenic fibrosing alveolitis. Thorax 1972; 27: 535-542

31 Thomeer MJ, Costabel U, Rizzato G et al. Comparison of registries of interstitial lung disease in three European countries. Eur Respir J 2001; 18, Suppl 32: $114-118$

32 Turner-Warwick M, Burrows B, Johnson A. Cryptogenic fibrosing alveolitis: clinical features and their influence on survival. Thorax 1980; 35: $171-180$

33 Yamaguchi M, Hosoda Y, Sasaki R et al. Epidemiological study on sarcoidosis in Japan. Recent trends in incidence and prevalence rates and changes in epidemiological features. Sarcoidosis 1989; 6: 138-146 